\title{
An audit cycle demonstrating improvement in delirium diagnosis, prevention and management (NICE guideline) at a district general hospital
}

\author{
Author: Wasim Khan
}

\begin{abstract}
Aims
To reassess if our hospital has improved its assessment of delirium diagnosis, prevention and management after raising awareness of delirium through education, training and dissemination of delirium care bundle throughout the trust.
\end{abstract}

\section{Methods}

A prospective sample of patients admitted to a district general hospital between 1 April 2017 to 8 April 2017 with confusion or developed confusion during their hospital stay. A total of 28 patients were identified and their notes were reviewed.

The National Institute for Health and Care Excellence (NICE) data collection tool was used to measure our practice in the diagnosis, prevention and management of delirium. The recommendations from initial audit were implemented and re-audited 6 months later.

\section{Results}

Initial audit showed that assessment of risk factors, indicators of delirium at presentation, assessment within 24 hours of admission and diagnosis (specialist clinical assessment) of delirium were below the NICE standards for delirium assessment. This led to recommendations to improve education and training of the junior medical staff along with implementation of delirium care bundle. Re-audit 6 months later showed improvement.

Standard 1 - initial assessment of risk factors for delirium: Re-audit showed improvement in assessment of all the domains especially assessing age and severe illness (Table 1).

\begin{tabular}{lll} 
Table 1. Standard 1 results & & \\
\hline Standard 1 - risk factors assessment & Initial audit & Re-audit \\
Age $>65$ years & $100 \%$ & $100 \%$ \\
Cognitive impairment & $66 \%$ & $87 \%$ \\
Current hip fracture & $30 \%$ & $68 \%$ \\
Severe illness & $83 \%$ & $100 \%$
\end{tabular}

Author: No affiliation provided

\section{Table 2. Standard 2 results}

$\begin{array}{lll}\begin{array}{l}\text { Standard } 2 \text { - indicators of delirium } \\ \text { at presentation }\end{array} & \text { Initial audit } & \text { Re-audit } \\ \text { Cognitive function } & 70 \% & 96 \% \\ \text { Perception } & 20 \% & 29 \% \\ \text { Physical function } & 66 \% & 96 \% \\ \text { Social behaviour } & 40 \% & 82 \%\end{array}$

Standard 2 - assessment of indicators of delirium at presentation: There was significant improvement in assessing indicators of delirium-like cognitive function, physical function and social behaviour but still need improvement in assessing perception (Table 2).

Standard 3 - assessment within 24 hours of admission: Assessment of hypoxia and infection were maintained to $100 \%$. The trend showed improvement in assessment of cognitive impairment, dehydration, immobility and pain. On the other hand, assessment of sleep disturbance, sensory impairment and poor nutrition showed decline and required improvement.

Standard 4-diagnosis (specialist clinical assessment) of delirium: Our re-audit has shown improvement in diagnosis (specialist clinical assessment) of delirium from $20 \%$ (initial audit) to $50 \%$ (reaudit) by using short-CAM (short confusion assessment method)/ the 4AT (rapid clinical instrument for delirium detection).

\section{Conclusion}

Significant improvement in delirium diagnosis, prevention and management was achieved through regular education and training of junior medical staff along with implementation of delirium care bundle. This led to improved patient care.

It has also raised the significance of falls assessment and polypharmacy in the trust.

\section{Conflict of interest statement}

There was no conflict of interest. 